\title{
Analysis of the Value of Tourism Resources of Intangible Cultural Heritage
}

\author{
Lanlan Liu',a, Qiang $Y u^{1, b}$ \\ ${ }^{1}$ Harbin University Harbin China \\ aliulanlan_001@163.com, byuqiang0708@163.com
}

Keywords: Intangible cultural heritage, Tourism resources, Analysis.

\begin{abstract}
Because of its non-renewable, scarcity and unique, intangible cultural heritage as a high quality tourist resource is conducive to enhancing the competitiveness and popularity of tourist destinations, and has a positive role in promoting the local social and cultural status. As the core value of the intangible cultural heritage, the cultural value of the intangible cultural heritage is to develop the cultural connotations of the intangible cultural heritage in the tourism development, and to develop the tourist products that meet the needs will be beneficial to the embodiment of its economic value, and will also provide the necessary economic support for the protection of the intangible cultural heritage. Therefore, in the development of intangible cultural heritage tourism, we should adhere to the principle of scientific, reasonable and moderate, use it in the protection, and further enrich its art science, history and social value, so that it can be more adaptable to the social development. However, there are many qualitative methods for evaluating tourism resources at present, because the excessive participation of subjective factors in the analysis process leads to the reduction of the results scientifically. In this paper, we will adopt the factor analysis method, in the process of analysis, by collecting the original variables of the analysis object, and selecting a few of the representative variables to empower objectively, and construct the data model to quantify the quantitative analysis. Thus, the objective analysis of the development value of the intangible cultural heritage tourism resources is realized.
\end{abstract}

\section{非物质文化遗产旅游资源价值分析 \\ 刘岗岗1, a, 于强1,b \\ 1哈尔滨学院, 哈尔滨, 中国 \\ aliulanlan_001@163.com, byuqiang0708@163.com}

关键词: 非物质文化遗产; 旅游资源; 分析

中文摘要. 非物质文化遗产因其不可再生性、稀缺性及唯一性, 作为优质的旅游资源, 有利 于增强旅游目的地的竞争力和知名度, 同时对提升当地的社会地位与文化地位有着积极的作 用。文化价值作为非物质文化遗产的核心价值, 在旅游开发中通过深入挖掘非物质文化遗产 的文化内涵, 开发符合需求的旅游产品, 将有利于体现其经济价值, 也将为非物质文化遗产 的保护提供必要的经济支撑。因此, 在非物质文化遗产旅游开发中, 要秉承科学、合理、适 度的原则, 在保护中利用, 在利用中进一步丰富它的艺术科学、历史、和社会价值, 使其更 加适应社会的发展。然而, 目前对于旅游资源的评价以定性分析的方法居多, 由于分析过程 中的主观因素参与过多致使价值评估的结果科学性降低。本文将采用因子分析法, 在分析过 
程中通过采集分析对象的原始变量, 并从中选取少数具有代表意义的变量客观赋权, 构建数 据模型时行量化分析, 从而, 实现了对非物质文化遗产旅游资源开发价值的客观分析的目的。

\section{1. 对非物质文化遗产价值分析的意义}

随着体验经济的到来，人们旅游的目的更加趋向于满足自身求异、怀旧、体验的心理需 求, 而非物质文化遗产本身作为地方传统文化的重要组成, 承载着民族的文化基因, 使特定 区域内人们适应自然和社会的智慧和审美情趣集中体现，而这恰恰能够满足旅游者领略民间 文化精髓, 体验地方文化, 了解地方文脉的需求。非物质文化遗产因其不可再生性、独特及 唯一性, 作为优质的旅游资源, 有利于增强旅游目的地的竞争力和知名度, 同时对提升当地 的社会地位与文化地位有积极的作用。对非物质文化遗产的旅游价值进行分析评价, 有利于 定量的研究非物质文化遗产的价值属性, 有利于区分资源的优劣, 确定开发顺序及开发规模, 对旅游业的正常发展有着重要的现实意义。

\section{2. 非物质文化遗产旅游价值分析模型的构建}

\section{1 指标采集表的设计}

非物质文化旅游资源定量研究是通过有代表意义的指标项目和指标体系进行评估和测定 的。通过定量地分析对非物质文化旅游资源的现状和特征进行分析、比较。通常情况下, 指 标越多越能全面地反映评价对象的状态, 在研究中受客观条件制约, 指标体系的构建也会受 到限制, 故构建指标体系时选择了更具针对性和代表性的非物质文化旅游资源的资源价值、 遗产质量情况、遗产开发环境条件、区位条件及其他条件诸多要素进行构建, 依据可统计性、 可获得性、可比较性、简明而科学地对哈尔滨市非物质文化旅游价值进行评价。具体指标体 系见表1。

表1＼cjkstart非物质文化旅游资源价值分析指标采集表

\begin{tabular}{|c|c|c|}
\hline 目标层 & 要素层 & 因子层 \\
\hline \multirow{5}{*}{$\begin{array}{l}\text { 非物质文化 } \\
\text { 遗产旅游资 } \\
\text { 源评价 (A) }\end{array}$} & 遗产资源价值（B1） & $\begin{array}{c}\text { 文化艺术价值(C1) } \\
\text { 历史价值(C2) } \\
\text { 审美价值(C3) } \\
\text { 观赏休賃价值(C4) } \\
\text { 其他价值(C5) }\end{array}$ \\
\hline & 遗产质量情况（ B2） & $\begin{array}{c}\text { 遗产规模(C6) } \\
\text { 遗产保护级别(C7) } \\
\text { 保存完整度(C8 } \\
\text { 真实性(C9) } \\
\text { 集聚组合状况(C10) }\end{array}$ \\
\hline & 遗产开发环境条件 ( B3) & $\begin{array}{c}\text { 政府政策 }(\mathrm{C} 11) \\
\text { 社区态度 }(\mathrm{C} 12) \\
\text { 经济发展速度及水平 }(\mathrm{C} 13) \\
\text { 地理环境 }(\mathrm{C} 14) \\
\text { 生态环境 }(\mathrm{C} 15)\end{array}$ \\
\hline & 区位条件（ B4） & $\begin{array}{c}\text { 区域可进入性(C16) } \\
\text { 外部交通的畅通性(C17) }\end{array}$ \\
\hline & 其他条件（ B5） & $\begin{array}{c}\text { 旅游服务水平(C18) } \\
\text { 现有旅游基础设施(C19) }\end{array}$ \\
\hline
\end{tabular}


该指标体系包含二级指标 5 个、三级指标 19 个。体系中指标的选择主要是依据统计年鉴所 公布的数据, 部分难以量化指标采取替代指标或问卷调查打分法。指标体系中将非遗资源价 值与遗产质量状况作为旅游环境研究的基础与核心, 非遗资源开发环境与区位等其他条件是 旅游环境可持续性的保证, 体现了非物质文化旅游开发必须要综合考虑社会经济、环境条件 等因素。

\section{2 数据处理}

因子分析法是通过对变量之间关系数据的计算进行统计的，在系统计算检验过程中，如 果相关系数矩阵的大部分相关系数小于 0.3 , 且在相关统计检验中未获通过, 则这些量将不适 用于因子分析方法。反映像相关矩阵、巴特利特球形检验、KMO检验适用于通过SPSS软件来 分析变量在因子分析中的适用性。

因子分析法的基本原理是将关系复杂的原始变量分为公共因子（适用于所有变量的共同 要素) 和特殊因子 (适用于单个变量的个性特征) 两个部分。原始变量与公共因子相比数量 较多, 但在统计计算中需要用公共因子来支撑和描述原始变量间的复杂关系。因此通过数量 较少的相互独立的因子变量来替代原始变量的复杂信息是该数学模型的根本出发点。假设原 有变量有 $\mathrm{p}$ 个, 分别用 $\mathrm{x} 1, \mathrm{x} 2, \mathrm{x} 3, \cdots, \mathrm{xp}$ 表示, 其中 $\mathrm{xi}(\mathrm{i}=1,2, \cdots, \mathrm{p})$ 是标准差为 1 , 均值为 0 的标 准化变量, F1, F2, F3, ․, Fm 是表示具有 $\mathrm{m}$ 个因子变量, 且 $\mathrm{m}<\mathrm{p}$ 。于是有:

$$
\begin{aligned}
& x_{1}=a_{11} F_{1}+a_{12} F_{2}+\cdots+a_{1 m} F_{m}+a_{1} \varepsilon_{1} \\
& x_{2}=a_{21} F_{1}+a_{22} F_{2}+\cdots+a_{2 m} F_{m}+a_{2} \varepsilon_{2} \\
& x_{p}=a_{p 1} F_{1}+a_{p 2} F_{2}+\cdots+a_{p m} F_{m}+a_{p} \varepsilon_{p}
\end{aligned}
$$

也可以表示成矩阵的形式;

$$
\mathrm{X}=A F+a \varepsilon
$$

其中, F用于表示公共因子或因子变量, 也可将其视为在高维空间彼此相交的 $\mathrm{m}$ 个坐标轴; 因子载荷矩阵用 $A$ 表示, 因子载荷用aij表示, 即为第 $\mathrm{i}$ 个原有变量在第 $\mathrm{j}$ 个因子变量上的负荷。 若将变量 $x i$ 和 $m$ 维因子空间中某个向量相对应, aij可视为标准回归系数, 即 xi在坐标轴Fj上 的投影。 $\varepsilon$ 可视为参差项, 即公共因子解释不了原有变量的部分。

本文对因子进行分析和指标的标准化处理选用的软件为 SPSS11.0。非物质文化旅游价值评 估模型的构建是由主成分分析法先确定出关键因子, 之后利用正交旋转法解释所得价值因子, 最终进行结果分析。依据数据指标的可获得性, 本文从19个指标中选取了 16 个指标来分析, 通过对 16 个操作指标的相关矩阵的计算, 相关系数大部分满足大于 0.3 的条件, 进行逐层主成 分分析是可行的。具体运算为从最后一层开始, 用主成分分析对每一分项的第一主成分确定 各指标权重, 再通过各自的权重乘以指标得分算出分项指数, 进而用主成分分析对得出的分 项指数值及第一主成分计算每一方面权重, 最后通过加权平均计算出非物质文化遗旅游价值 指数。依据方差总解释表显示, 特征根大于 1 的为前 3 个因子, 故这三个因子可作为主因子, 且包含原始变量 $86.426 \%$ 以上的信息。

表2.总方差

\begin{tabular}{|c|c|c|c|c|c|c|}
\hline & \multicolumn{3}{|c|}{ 初始特征值 } & \multicolumn{3}{|c|}{ 提取成分后特征值 } \\
\hline 主成分 & 特征值 & 解释方差比例 & $\begin{array}{c}\text { 累计解释方 } \\
\text { 差比例 }\end{array}$ & 特征值 & 解释方差比例 & $\begin{array}{c}\text { 累计解释方 } \\
\text { 差比例 }\end{array}$ \\
\hline 1 & 2.586 & 38.641 & 38.641 & 2.179 & 34.013 & 34.013 \\
\hline 2 & .972 & 24.302 & 67.963 & 2.014 & 27.238 & 61.250 \\
\hline 3 & .658 & 17.449 & 86.426 & 1.953 & 25.175 & 86.426 \\
\hline 4 & .584 & 4.608 & 91.417 & & & \\
\hline
\end{tabular}




\begin{tabular}{|l|l|l|l|l|l|l|}
\hline 5 & .399 & 3.552 & 95.296 & & & \\
\hline 6 & .310 & 2.842 & 97.657 & & & \\
\hline 7 & .184 & 1.160 & 98.997 & & & \\
\hline 8 & .108 & 1.001 & 100.00 & & & \\
\hline
\end{tabular}

表3. 主成分载荷矩阵

\begin{tabular}{|c|c|}
\hline & Component \\
\hline & 1 \\
\hline VAR00001 & 0.907 \\
\hline VAR00002 & 0.512 \\
\hline VAR00003 & 0.932 \\
\hline
\end{tabular}

依据成分提取矩阵结果, 能够得到 3 个主成分得分表达式, 具体为遗产资源价值为: 遗 产资源价值指数 $=0.288 * \mathrm{~F} 1+0.163 * \mathrm{~F} 2+0.296 * \mathrm{~F} 3$ 。同理, 依次可得出: 遗产质量情况指数 $=0.312 * \mathrm{~F} 1+0.292 * \mathrm{~F} 2+0.174 * \mathrm{~F} 3$; 遗产开发环境条件指数 $=0.118 * \mathrm{~F} 1+0.329 * \mathrm{~F} 2+0.256 * \mathrm{~F} 3$; 区位 条件指数 $=0.411 * \mathrm{~F} 1+0.198 * \mathrm{~F} 2+0.394 * \mathrm{~F} 3$ 。

将主因子贡献率视为权重可以计算出非物质文化旅游价值水平，具体表达为:

$$
\mathrm{F}=\sum \lambda_{\mathrm{i}} \mathrm{F}_{\mathrm{i}} / \sum \lambda_{\mathrm{i}}
$$

故F=F1*0.23434+F2*0.09185+F3*0.06680+F4*0.05569+F5*0.05000+ F6 * 0.04925 $+\mathrm{F} 7 * 0.04481+\mathrm{F} 8 * 0.03836$

\section{3. 结论}

本评价体系的构建不以任何非物质遗产为指定对象, 且在评分过程中以问卷随机发放的 形式, 故在操作过程中满足一般性要求。在向游客发放问卷时, 通过上述19个因子的问卷即 可客观地了解到非物质文化遗产的旅游价值水平。上述 19 个指标满分为 10 分, 合格为 5 分, 通 过计算即可得到非物质文化旅游综合和分项的平均值和理想值。所以本评价体系将适用于任 意非物质文化旅游价值的评价, 满足普遍适用性检验这一要求。

\section{致谢}

本文为哈尔滨市社科联重点调研项目《哈尔滨市非物质文化遗产旅游开发存在的问题及对 策分析》的阶段性成果之一。

\section{References}

[1] Ferdinand N., Williams N.L. International Festivals as Experience Production Systems,Tourism Management, vol.34,pp.202-210, 2013.

[2] Esteban Ruiz Ballesteros,Macarena Hernandez Ramirez, Identity and community the development of mining heritage tourism in Southern Spain, Tourism Management, vol.28,pp.677-687,2007.

[3] Susan O.Keitumetse, Sustainable Development and Cultural Heritage Management in Botswana : toward sustainable communities, Sustainable Developmen, vol. (5) ,pp.419-425, 2009.

[4] Meiyu Shi; Wei Zhu; Hongji Yang; Chen Li, Using agent-based model to simulate stakeholder balance model of tourism intangible cultural heritage, Multiagent and Grid Systems, vol.10.3233,pp. 91-103,2016. 